\title{
One-Year Clinical Outcome in End-Stage Heart Failure: Comparison of "High Urgent" Listing for Heart Transplantation with Mechanical Circulatory Support Implantation
}

\author{
Jakub Sunavsky ${ }^{1}$ Armin Zittermann ${ }^{1}$ Cenk Oezpeker ${ }^{1}$ Buntaro Fujita ${ }^{1}$ Uwe Fuchs ${ }^{1}$ \\ Jan F. Gummert ${ }^{1} \quad$ Uwe Schulz $^{1}$ \\ ${ }^{1}$ Department for Thoracic and Cardiovascular Surgery, Ruhr-University \\ Bochum, Bad Oeynhausen, Germany \\ Thorac Cardiovasc Surg 2015;63:647-652. \\ Address for correspondence Jakub Sunavsky, MD, Department for \\ Thoracic and Cardiovascular Surgery, Heart and Diabetes Center NRW, \\ Ruhr-University Bochum, Georgstrasse 11, 32545 Bad Oeynhausen, \\ Germany (e-mail: jsunavsky@hdz-nrw.de).
}

Abstract
Keywords
- end-stage heart
$\quad$ failure
- heart transplantation
- mechanical
$\quad$ circulatory support
- survival
- mortality
- high urgent listing

Background Heart transplantation (HTx) is still considered the therapeutic gold standard in end-stage heart failure.

Methods In "high urgent" (HU)-listed patients for HTx $(n=274)$ and patients receiving left ventricular assist device (LVAD) implants $(n=332)$, we compared 1-year overall survival (primary endpoint) and 1-year probability of HTx and therapy failure (the need for LVAD implantation in HU-listed patients or the need for HU listing in LVAD patients) (secondary endpoints).

Results In the HU and LVAD group, 1-year survival was 86.8 and $64.7 \%$, respectively $(p<0.001)$. The propensity score (PS)-adjusted hazard ratio of mortality did not differ between the groups and for the LVAD group (reference $=\mathrm{HU}$ group) was $=1.36$ (95\% confidence interval $[\mathrm{CI}]: 0.85-2.19 ; p=0.198)$. The PS-adjusted hazard ratio for the failure to receive $\mathrm{HTx}$ for the LVAD group (reference $=\mathrm{HU}$ group) was $=9.77(95 \% \mathrm{Cl}$ : $6.00-15.89 ; p<0.001)$. The corresponding hazard ratio for therapy failure for the LVAD group was $=0.16,95 \% \mathrm{Cl}: 0.10-0.27 ; p<0.001)$.

Conclusion Despite considerable differences in the probability of HTx and therapy failure, 1-year overall survival was similar in HU and LVAD patients.

\section{Introduction}

Orthotopic heart transplantation (HTx) continues to be the only curative therapeutic option for patients with end-stage heart failure, despite significant progress in the field of medical and resynchronization therapy, the development of surgical circulatory support devices, and promising experimental approaches in the field of regenerative medicine (e.g., stem cell research, tissue engineering). ${ }^{1}$ Unfortunately, however, there has been an imbalance between the number of patients listed for HTx and available donor hearts. ${ }^{2}$ In

received

March 5, 2015

accepted after revision

May 13, 2015

published online

July 15, 2015

Germany, for example, donor heart shortage increased by 25\% between 1997 and 2011. ${ }^{3}$

Currently, only $10 \%$ of all heart transplantations in Germany are performed in the "transplantable" (T) status, whereas 90\% are performed in the "high urgent" (HU) status. ${ }^{4}$ Patients in the latter status are critically ill ${ }^{5}$ and have very limited life expectancy if they do not receive HTx. Currently, the average waiting time for listed patients is 15 months, ${ }^{6}$ or 6 months if only HU-patients are considered. ${ }^{5}$ Because of donor heart shortage, the waiting time continues to rise. Consequently, mechanical assist devices are increasingly used

(c) 2015 Georg Thieme Verlag KG Stuttgart · New York
DOI http://dx.doi.org/ 10.1055/s-0035-1556816. ISSN 0171-6425. 
as a bridge to transplant (BTT). ${ }^{7}$ However, patients who are on mechanical circulatory support (MCS) have no allocation priority and can only be HU listed in case of MCS-related complications or MCS failure. Therefore, it remains controversial whether or not MCS is able to improve survival in HTx candidates. ${ }^{8}$ Because of the poor clinical outcome in patients with biventricular assist device (BVAD) or total artificial heart (TAH) implants, ${ }^{9}$ the current strategy is to prefer left ventricular assist device (LVAD) implants and, if necessary, to perform extracorporeal membrane oxygenation (ECMO).

This study, therefore, aimed to compare clinical outcomes of patients with end-stage heart failure who received LVAD implants as BTT and those who were HU listed for HTx.

\section{Methods}

\section{Patients}

For this retrospective single-center study, 791 consecutive end-stage heart failure patients aged $>15$ years who were admitted to our institution from November 2005 to November 2012 (-Fig. 1) were considered. Of these 791 patients, 274 patients were HU listed (designated HU group) and 517 patients received MCS implants. We excluded 82 patients with MCS support from data analysis because they initially received destination therapy. Additional 103 patients were excluded because they had BVAD or TAH implants, leaving 332 patients with LVAD implants who were included in the data analysis (designated LVAD group). In accordance with Eurotransplant, ${ }^{10}$ the following criteria were used for HU listing: inotropic support therapy with dobutamine $>7.5$ $\mu \mathrm{g} / \mathrm{kg} / \mathrm{min}$ or milrinone $>0.5 \mu \mathrm{g} / \mathrm{kg} / \mathrm{min}$ for at least 48 hours, venous oxygen saturation $\left(\mathrm{SVo}_{2}\right)<55 \%$, cardiac index $<2.2$ $\mathrm{L} / \mathrm{min} / \mathrm{m}^{2}$, pulmonary capillary wedge pressure $>10 \mathrm{~mm} \mathrm{Hg}$, sodium $<136 \mathrm{mmol} / \mathrm{L}$, creatinine increase despite maximal heart failure treatment, increase in transaminase concentrations, or cerebral perfusion deficit. In line with international recommendations, ${ }^{11}$ patients received LVAD implants as a BTT in case of failing maximal medical, surgical, and/or pacemaker/defibrillator therapies and high risk of dying before receiving HTx. The decision for HU listing or LVAD

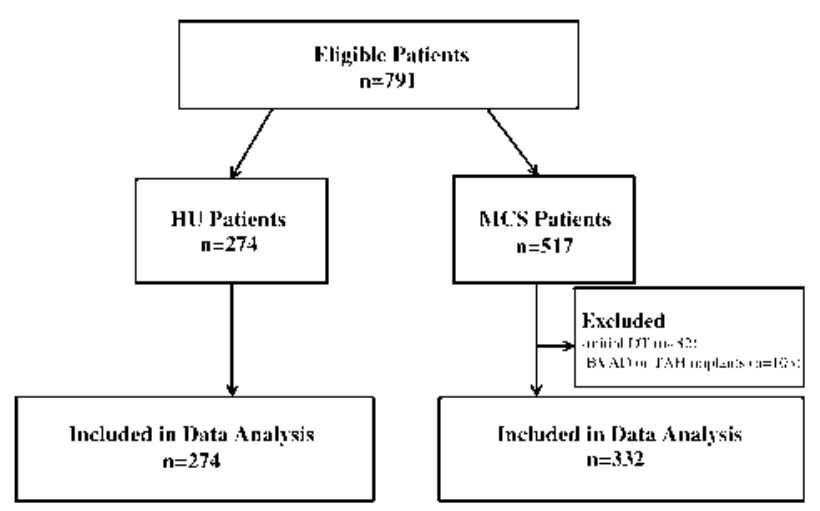

Fig. 1 Flowchart of included patients. BVAD, biventricular assist device; DT, destination therapy; HU, high urgent; MCS, mechanical circulatory support; TAH, total artificial heart. implants was made in weekly institutional and interdisciplinary expert conferences.

\section{Endpoints}

Primary endpoint was overall survival within 1-year of HU listing or LVAD implantation. Secondary endpoints were the probability of HTx and freedom from therapy failure (the need for MCS implantation in the HU group or the need for HU listing in the LVAD group) during follow-up.

\section{Statistical Analysis}

Continuous data are presented as mean values and standard deviation (SD) or median and interquartile range when appropriate. Normal distribution of data was tested using the Kolmogorov-Smirnov test and was assumed if $p$-values were $>0.05$. We used the Student $t$-test and the MannWhitney $U$ test to test for differences between the groups. Categorical data are expressed as numbers (percentage) and the chi-square test was performed to test for differences between the groups. The probability of 1-year survival and event-free survival was calculated using Kaplan-Meier estimates as a function of time. The log-rank test was used to test for statistical differences between the groups. The statistical analyses were performed according to the intention-to-treat principle. Some patients in the HU and LVAD groups were removed from the transplant list because of significant improvement or worsening of the clinical status, switch to destination therapy, or other reasons, such as malignancy, psycho-social instability, drug and nicotine or alcohol abuse. These patients were censored at the time the event occurred. We also performed propensity score (PS)-adjusted Cox regression analysis to evaluate the association of the study groups with 1-year mortality and secondary endpoints. Data are expressed as hazard ratio (HR) with 95\% confidence interval $(\mathrm{CI})$. The PS-adjusted analyses were performed to control for selection bias due to the result of nonrandom group assignment. The PS derivation model was constructed using multivariable logistic regression, with study group as the binominal dependent variable and the preoperative anthropometric and clinical variables listed in - Table $\mathbf{1}$ as predictor variables. The model's reliability and predictive ability were measured using the Hosmer-Lemeshow test and the c-index, respectively.

$p$-values $<0.05$ were considered statistically significant. All statistical analyses were performed using IBM SPSS Statistics, version 20 (IBM Corp., Armonk, New York, United States).

\section{Results}

\section{Baseline Characteristics}

Baseline characteristics are summarized in - Table 1, broken down by study group. In detail, the vast majority of patients were Caucasians. Groups were comparable regarding systolic pulmonary pressure and the prevalence of previous cardiac surgery. However, age, weight, heart rate, percentage of males, and prevalence of INTERMACS level 1 or 2 were significantly higher, and systolic arterial pressure and 
Table 1 Baseline characteristics of the study groups

\begin{tabular}{|c|c|c|c|}
\hline & $\mathrm{HU}(n=274)$ & $\operatorname{LVAD}(n=332)$ & p-Value \\
\hline Race, Caucasians ( $N, \%)$ & $271(98.9 \%)$ & $434(99.8 \%)$ & 0.27 \\
\hline Age $(y)$ & $49 \pm 13$ & $57 \pm 13$ & $<0.001$ \\
\hline Gender, males $(N, \%)$ & $223(81.4 \%)$ & $293(88.3 \%)$ & 0.021 \\
\hline Systolic pulmonary artery pressure $(\mathrm{mm} \mathrm{Hg})$ & $42 \pm 15$ & $41 \pm 14$ & 0.199 \\
\hline Weight (kg) & $75 \pm 14$ & $80 \pm 17$ & $<0.001$ \\
\hline Heart rate $(N)$ & $79(68-91)$ & $92(80-106)$ & $<0.001$ \\
\hline Systolic arterial pressure $(\mathrm{mm} \mathrm{Hg})$ & $104(92-115)$ & $92(81-106)$ & $<0.001$ \\
\hline Left ventricular ejection fraction (\%) & $22(20-28)$ & $20(15-25)$ & $<0.001$ \\
\hline Previous cardiac surgery $(N, \%)$ & $52(19.0 \%)$ & $86(25.9 \%)$ & 0.082 \\
\hline \multicolumn{4}{|l|}{ Diagnosis } \\
\hline Ischemic cardiomyopathy $(N, \%)$ & $133(48.5 \%)$ & $132(39.8 \%)$ & 0.033 \\
\hline Dilated cardiomyopathy $(N, \%)$ & $87(31.8 \%)$ & 181 (54.5\%) & $<0.001$ \\
\hline Others $(N, \%)$ & $54(19.7 \%)$ & $19(5.7 \%)$ & $<0.001$ \\
\hline \multicolumn{4}{|l|}{ Concomitant diagnoses } \\
\hline INTERMACS level $1+2(N, \%)$ & 59 (21.5\%) & 187 (56.3\%) & $<0.001$ \\
\hline INTERMACS level $3(N, \%)$ & $214(78.1 \%)$ & $123(37.0 \%)$ & $<0.001$ \\
\hline INTERMACS level $4+5(N, \%)$ & $1(0.4 \%)$ & $22(6.6 \%)$ & $<0.001$ \\
\hline Diabetes mellitus $(N, \%)$ & $58(21.2 \%)$ & 99 (29.9\%) & 0.016 \\
\hline Pulmonary artery hypertension ( $N, \%)$ & $218(79.6 \%)$ & $182(55.0 \%)$ & $<0.001$ \\
\hline Infection $(N, \%)$ & $60(21.9 \%)$ & $130(39.3 \%)$ & $<0.001$ \\
\hline \multicolumn{4}{|l|}{ Biochemical parameters } \\
\hline Sodium (mmol/L) & $136 \pm 5$ & $135 \pm 6$ & 0.377 \\
\hline Hemoglobin $(\mathrm{g} / \mathrm{L})$ & $12.1(10.5-13.4)$ & $10.9(10.1-12.3)$ & $<0.001$ \\
\hline Leukocytes $\left(\mathrm{N}, 10^{3} / \mathrm{L}\right)$ & $7.6(6-9.5)$ & $9.2(7.2-12.6)$ & $<0.001$ \\
\hline Urea (mg/L) & $54(40-86)$ & $66(43-95)$ & $<0.001$ \\
\hline Creatinine (mg/L) & $1.3(0.95-1.7)$ & $1.4(1.0-2.0)$ & $<0.001$ \\
\hline Total cholesterol (mg/L) & $170 \pm 56$ & $158 \pm 52$ & 0.004 \\
\hline Uric acid $(\mathrm{mg} / \mathrm{L})$ & $6.7 \pm 2.5$ & $6.9 \pm 3.1$ & 0.336 \\
\hline Blood glucose $(\mathrm{mg} / \mathrm{L})$ & $101(91-116)$ & $110(93-135)$ & $<0.001$ \\
\hline Bilirubin (mg/L) & $0.87(0.59-1.32)$ & $1.14(0.74-2.13)$ & $<0.001$ \\
\hline \multicolumn{4}{|l|}{ Support } \\
\hline Intra-aortic balloon pump ( $N, \%)$ & $84(30.7 \%)$ & $119(36.0 \%)$ & 0.194 \\
\hline Mechanical ventilatory support $(N, \%)$ & $26(9.5 \%)$ & 65 (19.6\%) & $<0.001$ \\
\hline Extracorporeal membrane oxygenation ( $N, \%)$ & $15(5.5 \%)$ & 47 (14.2\%) & $<0.001$ \\
\hline Dialysis $(N, \%)$ & $58(21.2 \%)$ & $65(19.6 \%)$ & 0.685 \\
\hline Pacemaker/defibrillator implants $(N, \%)$ & $217(79.2 \%)$ & $217(65.6 \%)$ & $<0.001$ \\
\hline
\end{tabular}

Abbreviations: HU, high urgent; LVAD, left ventricular assist device.

ejection fraction significantly lower in the LVAD group than in the HU group. In addition, the prevalence of dilated cardiomyopathy and infection was higher in the LVAD group than in the HU group. The high prevalence of infections in both the groups was because of poor hemodynamic condition leading to congestive pneumonia and other secondary infections. Most biochemical parameters differed significantly between the groups, with the exception of sodium and uric acid. The LVAD group needed more ECMO implants, more mechanical ventilatory support, but less pacemaker or defibrillator support than the HU group.

The PS ranged from a low of 0.03495 to a high of 1.0000 . The PS model was reliable (Hosmer-Lemeshow test, $p=0.094$ ) and discriminate (c-statistic $=0.88,95 \% \mathrm{CI}$ : 0.86-0.91). 
In the LVAD group, the majority of patients received HeartMate II (Thoratec Corp., Peasanton, California, United States; $n=131)$ and HeartWare (HVAD, HeartWare International Inc. Framingham, Massachusetts, United States; $n=95$ ) implants. The remaining LVAD patients received DuraHeart (Terumo Heart Inc., Ann Arbor, Michigan, United States; $n=52$ ), Ventracor (Ventracor Ltd., Chatswood, New South Wales, Australia; $n=40)$, and others $(n=14)$.

During follow-up, three patients in the HU group were censored because of good condition, such as improvement of hemodynamics or reduction of inotropic support, and three patients because of bad condition, such as increased lactate levels or need for vasopressor therapy. In the LVAD group, patients were censored because of bad condition $(n=15)$, destination therapy ( $n=13$ ) because of contraindication for HTx, such tumor diagnosis or irreversible pulmonary hypertension, and other reasons $(n=6)$, such as psychosocial instability, alcohol or drug abuse.

\section{Primary Endpoints}

Unadjusted 1-year overall survival was $86.8 \%$ in the HU group and $64.7 \%$ in the LVAD group ( $p<0.001$ ). The PS-adjusted HR of 1-year mortality is illustrated in - Fig. 2 and, with the HU group as reference group, for the LVAD group was $=1.36(95 \%$ CI: $0.85-2.19 ; p=0.198)$. In sensitivity analysis, we compared 1-year survival in the HU group with the entire MCS group (332 LVAD, 49 BVAD, and 54 TAH patients, see - Fig. 1). The PS-adjusted HR of 1-year mortality, with the HU group as reference group, for the entire MCS group was $=1.68(95 \% \mathrm{CI}$ : $1.08-2.62 ; p=0.022$ ). In the MCS group, 1-year survival in patients with implants of LVADs, BVADs, and TAHs was 64.7, 40.1 , and $34.4 \%$, respectively $(p<0.001)$.

\section{Secondary Endpoint}

Unadjusted 1-year probability of HTx was $79.5 \%$ in the HU group and $20.5 \%$ in the LVAD group $(p<0.001)$. The PSadjusted HR of the failure to receive HTx is presented in - Fig. 3 and, with the HU group as reference group, for the LVAD group was $=9.77$ (95\% CI: 6.00-15.89; $p<0.001)$.

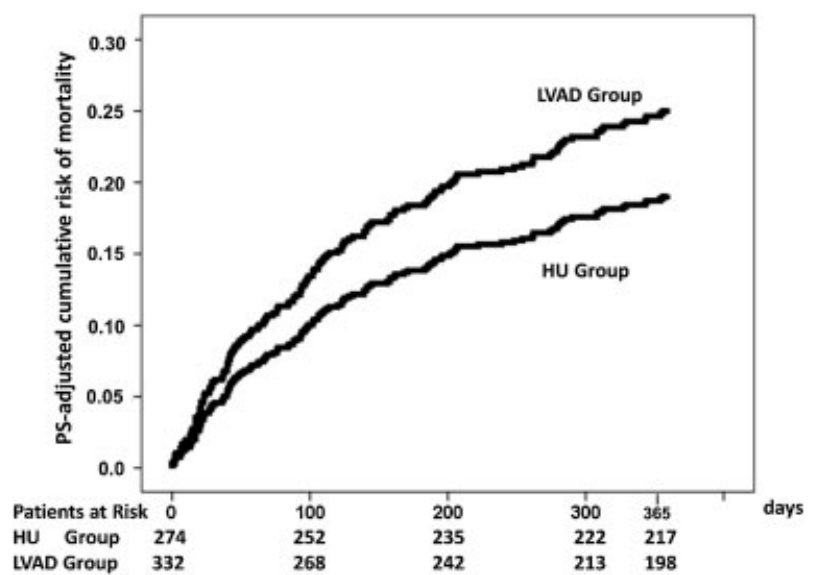

Fig. 2 Propensity score (PS)-adjusted cumulative incidence of 1-year overall mortality, broken down by study group. HU, high urgent; LVAD, left ventricular assist device.

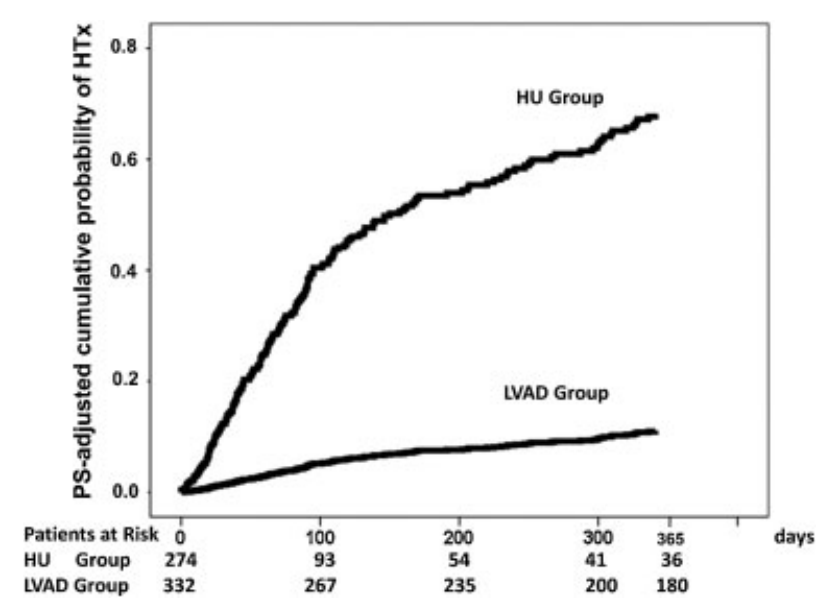

Fig. 3 Propensity score (PS)-adjusted cumulative incidence of the probability of heart transplantation, broken down by study group.

In the HU group, 199 patients were transplanted and 6 died during follow-up. In the LVAD group, 26 patients were transplanted of whom 5 patients died during follow-up. Unadjusted freedom from therapy failure was $78.1 \%$ in the HU group and $88.6 \%$ in the LVAD group ( $p=0.016$ ). The PS-adjusted HR for therapy failure was markedly lower in the LVAD group than in the $\mathrm{HU}$ group $(\mathrm{HR}=0.16,95 \% \mathrm{CI}$ : $0.10-0.27$; $p<0.001$ ) (-Fig. 4). In the HU group, 57 patients received MCS implants (35 LVAD, 16 BVAD, and 6 TAH implants) of whom 17 patients died during follow-up. In the LVAD group, 38 patients were $\mathrm{HU}$ listed of whom 10 patients died during follow-up.

\section{Discussion}

To the best of our knowledge, this is the first study comparing clinical outcomes of HU-listed patients with patients receiving LVAD support in end-stage heart failure. The principal finding of this study is that within 1 year of HU listing or LVAD implantation, PS-adjusted mortality did not differ

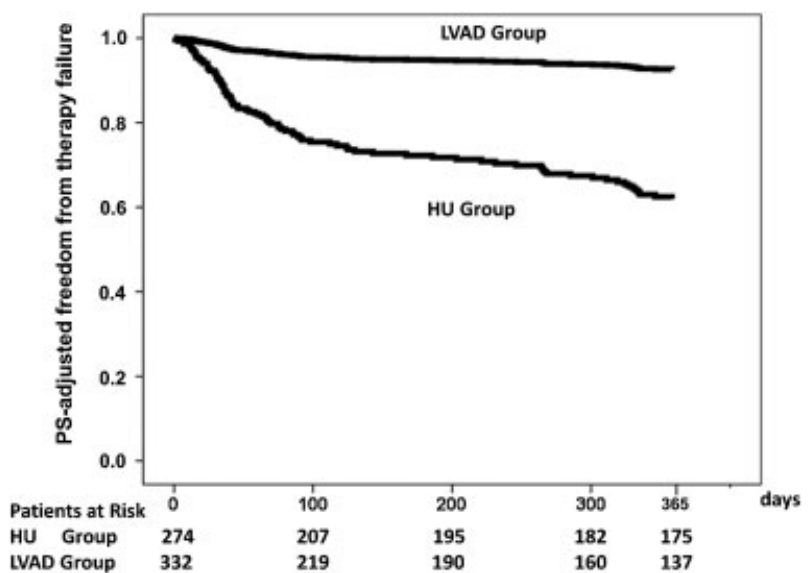

Fig. 4 Propensity score (PS)-adjusted incidence of freedom from therapy failure, broken down by study group. HU, high urgent; LVAD, left ventricular assist device. 
significantly between the groups. Moreover, compared with the LVAD group, PS-adjusted probability of HTx was markedly higher in the HU group, whereas PS-adjusted therapy failure was significantly higher in the HU group than in the MCS group.

LVAD implantation is an important treatment option for improving survival in patients with end-stage heart failure. ${ }^{12,13}$ Therefore, LVAD implants are increasingly used as a BTT. ${ }^{7}$ In the HU group of our study, $21 \%$ of the patients were switched to LVADs and other MCSs and this opportunity was certainly crucial for acceptable 1 -year survival. Stabilization of the hemodynamic condition by LVAD implantation may also have contributed to the similar 1-year survival of the LVAD group compared with the HU group, despite the considerably higher probability of HTx in the latter group. It is, however, also noteworthy that we did not study long-term clinical outcomes. Currently, 5-year survival is less than 50\% in patients with LVAD implants, ${ }^{9}$ whereas in HTx patients median survival exceeds 10 years. ${ }^{14}$ Therefore, it is important that a clinic can offer both HTx and MCS implants. Results of our sensitivity analysis support the strategy of using BVAD or TAH implants very restrictively, as 1-year survival of the MCS group worsened considerably by including patients with BVAD and TAH implants into the statistical analysis.

The problem with the current allocation system in Europe is that, because of donor heart shortage and long waiting times, most patients with INTERMACS levels 1 and 2 need LVAD implants as a BTT. However, patients in HU status are prioritized over T-listed and LVAD patients without complications. ${ }^{15}$ Currently, patients in Germany without HU status hardly have any chance of receiving HTx. In our study, transplant outcome was excellent and only relatively few LVAD patients were forwarded to HTx. Therefore, it is our clinical strategy to delay LVAD implantation for as long as possible, thereby hoping that the probability of receiving a donor heart in HU status would increase. Hence, it is our policy to prefer urgent HTx and to limit LVAD implantation to those patients who would otherwise inevitably develop cardiogenic shock and multiorgan failure. Our results show that, when following this strategy, patients primarily listed as HU have similar survival outcomes compared with patients primarily receiving LVAD, despite the HTX-associated complication risks and the higher chances of therapy failure. In 2008 , the probability of receiving a donor heart within 1 year was $25 \%$ for LVAD patients in Europe. ${ }^{16}$ Our findings of $21 \%$ HTx probability in LVAD patients are comparable with the earlier results. In line with our data, Uriel et al $^{7}$ have demonstrated that in LVAD patients LVAD-related complications/ malfunctions comprise the majority of transplant recipients.

It has been suggested that despite improved technology, the prognosis after LVAD implantation is not comparable with that after HTx. ${ }^{17}$ Although our data do not support this hypothesis with regard to 1-year survival, the current strategy of LVAD implantation as a BTT needs to be reconsidered. The solution might be the new Eurotransplant heart allocation policy. The new Cardiac Allocation Score is based on different risk score models: the Seattle Heart Failure Model (SHFM) and the Index for Mortality Prediction after Cardiac
Transplantation (IMPACT) score. SHFM and IMPACT should provide accurate risk stratification for urgency of HTx, as well as success after HTx. ${ }^{1}$ In general, patients with a high risk of dying without a transplant are prioritized, but only if they have an expected survival after HTx that exceeds the expected survival on the waiting list. ${ }^{4}$

Besides a new heart allocation policy, donor heart shortage also needs to be urgently addressed. In 2013, heart donation rates per million population (PMP) were nearly twice as high in Austria (7.6 PMP) and Belgium (6.7 PMP), compared with those in Germany (3.9 PMP) and the Netherlands (2.2 PMP). ${ }^{6}$ Notably, organ donation rates have nearly doubled in Belgium within 2 years after the implementation of the new law in $1987 .^{18}$ The legislative system for organ donation has been analyzed in several European countries. ${ }^{18}$ Type of legislation was an independent factor of differences in donation rates between countries. However, others ${ }^{19}$ have also pointed to the multifactorial character of the donation process. According to their conclusion, national legislations alone are unlikely to explain variations in donation rates between countries. A combination of legislation, potential of medically suitable donors, investments in health care and infrastructure, underlying public attitudes, religion, and education may play a role. $^{20}$

This study has some limitations. First, it is a retrospective investigation. Even PS-adjustment cannot definitively rule out that unidentified factors have influenced study outcomes. Second, results are only valid for mid-term survival. Third, our LVAD patients were only eligible for HU status when lifethreatening complications occurred. This situation has profound consequences on the chance of receiving HTx.

In conclusion, our data indicate similar 1-year survival in HU-listed patients and patients undergoing LVAD implants as a BTT. In addition, the probability of receiving HTx is higher and freedom from therapy failure is lower in HU-listed patients than in LVAD patients. Data support the assumption that a new HTx allocation system and campaigns to reduce donor organ shortage are needed.

\section{References}

1 Bernhardt AM, Rahmel A, Reichenspurner $\mathrm{H}$. The unsolved problem of organ allocation in times of organ shortage: the German solution? J Heart Lung Transplant 2013;32(11):1049-1051

2 Ketchum ES, Moorman AJ, Fishbein DP, et al. Predictive value of the Seattle Heart Failure Model in patients undergoing left ventricular assist device placement. J Heart Lung Transplant 2010;29(9): 1021-1025

3 Smits JM. Actual situation in Eurotransplant regarding high urgent heart transplantation. Eur J Cardiothorac Surg 2012;42(4):609-611

4 Smits JM, de Vries E, De Pauw M, et al. Is it time for a cardiac allocation score? First results from the Eurotransplant pilot study on a survival benefit-based heart allocation. J Heart Lung Transplant 2013;32(9):873-880

5 Frazier OH, Rose EA, Oz MC, et al. HeartMate LVAS Investigators. Multicenter clinical evaluation of the HeartMate vented electric left ventricular assist system in patients awaiting heart transplantation. J Thorac Cardiovasc Surg 2001;122:186-195

6 Anonymous. Eurotransplant: donation, waiting lists, and transplants. In: Oosterlee A, Rahmel A, eds. Annual Report 2013 of the 
Eurotransplant International Foundation. Leiden, the Netherlands: Eurotransplant International Foundation; 2013. Available at: www. eurotransplant.org/cms/index.php?page=annual_reports. Accessed November 5, 2014

7 Uriel N, Jorde UP, Woo Pak S, et al. Impact of long term left ventricular assist device therapy on donor allocation in cardiac transplantation. J Heart Lung Transplant 2013;32(2):188-195

8 Nativi JN, Drakos SG, Kucheryavaya AY, et al. Changing outcomes in patients bridged to heart transplantation with continuous- versus pulsatile-flow ventricular assist devices: an analysis of the registry of the International Society for Heart and Lung Transplantation. J Heart Lung Transplant 2011;30(8):854-861

9 Kirklin JK, Naftel DC, Pagani FD, et al. Sixth INTERMACS annual report: a 10,000-patient database. J Heart Lung Transplant 2014; 33(6):555-564

10 Anonymus. ET Thoracic Allocation System (EThAS). Chapter 6. Eurotransplant Manual - version 3.3; July 10, 2014-subject to change. Available at: http://www.eurotransplant.org/cms/mediaobject. php?file=Chapter6_Thoracic22.pdf Accessed November 15, 2014

11 Peura JL, Colvin-Adams M, Francis GS, et al; American Heart Association Heart Failure and Transplantation Committee of the Council on Clinical Cardiology; Council on Cardiopulmonary, Critical Care, Perioperative and Resuscitation; Council on Cardiovascular Disease in the Young; Council on Cardiovascular Nursing; Council on Cardiovascular Radiology and Intervention, and Council on Cardiovascular Surgery and Anesthesia. Recommendations for the use of mechanical circulatory support: device strategies and patient selection: a scientific statement from the American Heart Association. Circulation 2012;126(22):2648-2667

12 Rose EA, Gelijns AC, Moskowitz AJ, et al; Randomized Evaluation of Mechanical Assistance for the Treatment of Congestive Heart
Failure (REMATCH) Study Group. Long-term use of a left ventricular assist device for end-stage heart failure. N Engl J Med 2001; 345(20):1435-1443

13 Attisani M, Centofanti P, La Torre M, et al. Advanced heart failure in critical patients (INTERMACS 1 and 2 levels): ventricular assist devices or emergency transplantation? Interact Cardiovasc Thorac Surg 2012;15(4):678-684

14 Lund LH, Edwards LB, Kucheryavaya AY, et al. The Registry of the International Society for Heart and Lung Transplantation: thirtieth official adult heart transplant report-2013; focus theme: age. J Heart Lung Transplant 2013;32(10): 951-964

15 Anonymous: Donation, waiting lists, and transplants. In: Oosterlee A, Rahmel A, eds. Annual Report 2010 of the Eurotransplant International Foundation. Leiden, The Netherlands: Eurotransplant International Foundation; 2010:34-47

16 Strüber M, Sander K, Lahpor J, et al. HeartMate II left ventricular assist device; early European experience. Eur J Cardiothorac Surg 2008;34(2):289-294

17 Kamiya H, Koch A, Sack FU, et al. Who needs 'bridge' to transplantation in the presence of the Eurotransplant high-urgency heart transplantation program? Eur J Cardiothorac Surg 2008;34(6): 1129-1133, discussion 1134-1135

18 Gimbel RW, Strosberg MA, Lehrman SE, Gefenas E, Taft F. Presumed consent and other predictors of cadaveric organ donation in Europe. Prog Transplant 2003;13(1):17-23

19 Gevers S, Janssen A, Friele R. Consent systems for post mortem organ donation in Europe. Eur J Health Law 2004;11(2):175-186

20 Roels L, Spaight C, Smits J, Cohen B. Donation patterns in four European countries: data from the donor action database. Transplantation 2008;86(12):1738-1743 